\title{
Cirripedios en la dieta del molusco herbívoro Chiton granosus Frembly, 1827 (Mollusca, Placophora) presente en el intermareal rocoso de Iquique, norte de Chile
}

\author{
Moisés A. Aguilera \\ Departamento de Ecología, Pontificia Universidad Católica de Chile \\ Casilla 114-D, Santiago, Chile
}

RESUMEN. Se entrega información preliminar cuantitativa, sobre la presencia de cirripedios en estados de larvas cypris y estados post-metamórficos en la dieta del molusco Chiton granosus Frembly, 1827 (Mollusca, Placophora) recolectado en Iquique, Chile. En diciembre de 2000, se observó la presencia de larvas cypris y post-metamórfico con una importante abundancia relativa porcentual $(\mathrm{N}=30,54,1$ y $39,9 \%$, respectivamente) y con una frecuencia de ocurrencia del 100\% en el total de estómagos. Además, se registró una alta frecuencia de larvas cypris $(\mathrm{F} \%=67)$ en la porción final del intestino, de éstas 15 presentaron signos de actividad vital (movimiento de apéndices) en el $45 \%$ del total de estómagos analizados. La ingesta de cirripedios en estados cypris y post-metamórficos, fue observada también con una alta frecuencia durante cinco meses de estudio $(\mathrm{N}=10,96 \% \pm 6,5$ y $78 \% \pm 23,6)$.

Palabras clave: Chiton granosus, dieta, cirripedios, larvas cypris, post-metamórficos, omnivoría.

\section{Barnacles in the diet of the molluscan grazer Chiton granosus Frembly, 1827 (Mollusca, Placophora) present on the intertidal rocky shore of Iquique, northern Chile}

\begin{abstract}
Preliminary quantitative information is given on the presence of barnacle cyprids and post-metamorphic stages in the diet of the molluscan grazer Chiton granosus Frembly 1827 (Mollusca: Placophora) sampled at Iquique, Chile. In December 2000, we observed the presence of barnacle cyprids and post-metamorphic stages at a high relative percentage of abundance ( $\mathrm{N}=30,54,1 \%$ and $39,9 \%$, respectively) and they were frequent in all of the individuals analyzed. Moreover, a high frequency of ocurrence of cyprid larvae $(\mathrm{F} \%=67)$ was recorded in the distal portion of the intestine; of these larvae, 15 were observed with signs of vital activity (appendage movement) in $45 \%$ of all the stomachs analyzed. Ingestion of barnacle cyprids and post-metamorphic stages was observed at a high frequency throughout the five months of the study $(\mathrm{N}=10,96 \% \pm 6,5$ and $78 \% \pm 23,6)$.
\end{abstract}

Key words: Chiton granosus, diet, barnacles, cyprid larvae, post-metamorphic larvae, omnivory.

Se ha observado en sistemas intermareales que algunos moluscos herbívoros ingieren en forma regular hydroides, briozoos y cirripedios (chitones Kaas \& van Belle, 1985; Patella vulgata Hill \& Hawkins, 1991), y que de esta forma pueden tener efectos significativos sobre su distribución y abundancia (cirripedios Acmea spp. Dayton, 1971; Collisella grata Chan \& Williams, 2003). En este sentido, en la zona de Mehuín en el sur de Chile, Jara (1980, fide Moreno \& Jaramillo, 1983) observó que el molusco herbívoro Chiton granosus (Frembly) ingería en forma selectiva larvas cypris de cirripedio. Según Jara (1980, fide Moreno \& Jaramillo, 1983) estas larvas serían el alimento principal de $C h$. 
granosus, pero otros estudios sobre la dieta de esta especie realizados en la zona central de Chile no han señalado este tipo de ingesta (Otaíza, 1986; Santelices et al., 1986).

En este documento, se presenta información cuantitativa preliminar sobre la ingesta de cirripedios por parte del molusco herbívoro Ch. granosus, que puede ser de importancia en el conocimiento sobre la historia natural de esta especie y también en la comprensión del efecto que tendría en la estructura comunitaria intermareal.

El estudio sobre la dieta de Ch. granosus, se realizó en el sector de la Península Cavancha, Iquique $\left(22^{\circ} 13^{\prime} \mathrm{S}-70^{\circ} 27^{\prime} \mathrm{W}\right)$. Para esto se recolectaron al azar, durante marea baja, 30 individuos de tallas entre 5,5 y $15,7 \mathrm{~cm}$ (ancho medido al nivel de la $5^{\text {ta }}$ placa) en diciembre de 2000, y un mínimo de 10 cada dos meses entre febrero y octubre de 2001. Primero los individuos fueron disectados in situ en su parte posterior con un bisturí, sin alterar el tejido estomacal, así se registró la presencia de larvas cypris y de otros invertebrados con signos de actividad vital presente en la parte posterior del intestino. Seguidamente, a los individuos se les inyectó una solución de formalina al $10 \%$ para detener totalmente el proceso de digestión estomacal (Santelices et al., 1986). Una vez en el laboratorio, se cuantificó la frecuencia de ocurrencia porcentual de cada ítem en el total de estómagos (F\%) que es la proporción de individuos donde se registró cada ítem. Para los individuos recolectados en diciembre 2000, también se realizó la estimación del porcentaje numérico de cada ítem en el total de estómagos (abundancia relativa porcentual), mediante la cuantificación de éstos en una placa Petri graduada con 30 puntos de intersección, cuyos valores se expresaron en términos porcentuales. Además, se realizó la identificación de los ítemes y restos de algas e invertebrados presentes en la porción final del intestino (bolos fecales a punto de ser expulsados).

En el contenido estomacal de Ch. granosus durante diciembre de 2000, se observó una amplia variedad de ítemes tanto de tipo vegetal como animal. Estos últimos correspondieron principalmente a larvas cypris de cirripedio indeterminado y estados post-metamórficos del cirripedio Jehlius cirratus, larvas de insecto quironómido y moluscos en estado post-metamórfico (principalmente Nodilittorínidos). Los cirripedios (cypris + estados postmetamórficos) se registraron con una abundancia relativa porcentual de 94,0\% la cual fue alta en comparación a los otros ítemes ingeridos (Fig. 1), y además fueron observados en el 100\% (F\%) de los individuos analizados. De estos estados, las larvas cypris se presentaron con una mayor abundancia (16,6 cypris/individuo $\pm 9,8)$, que los postmetamórficos (11,9 post-metamórficos/individuo \pm 8,4) (Fig. 2).

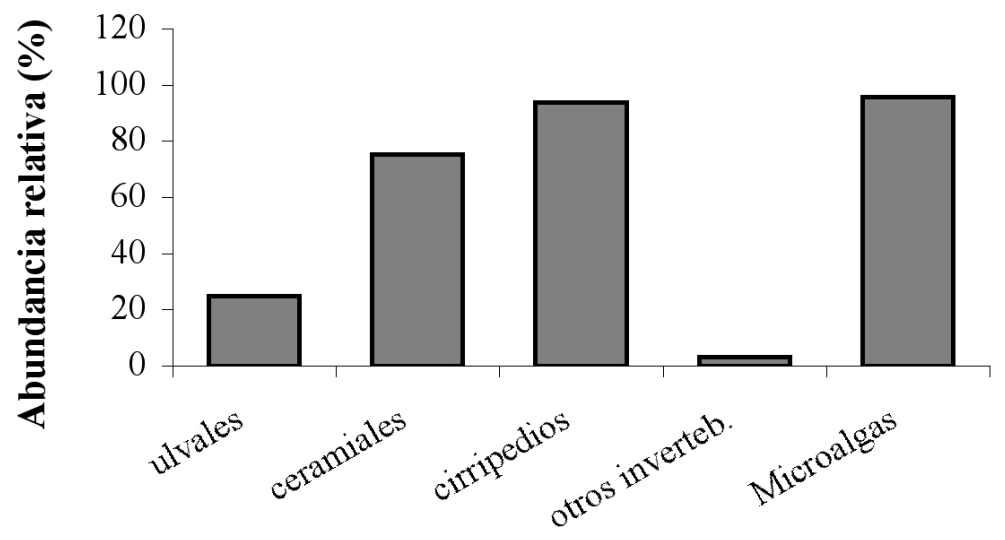

Figura 1. Abundancia relativa porcentual $(\mathbf{N}=30)$ estimada en 30 puntos de intersección, de los ítemes más importantes observados en el contenido estomacal de Chiton granosus recolectado en diciembre de 2000 en el sector de Península Cavancha, Iquique.

Figure 1. Percentual relative abundance $(N=30)$ estimated in 30 intersection points, of the more important items observed in the gut content of Chiton granosus collected in December 2000 in Peninsula Cavancha, Iquique. 


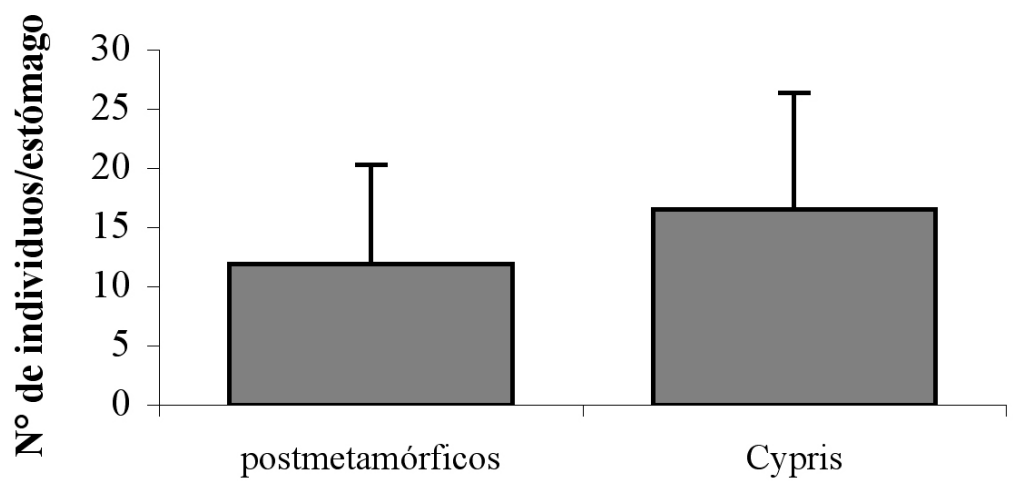

Figura 2. Abundancia promedio ( $\pm \mathrm{DS} ; \mathbf{N}=30$ ) de larvas cypris y estados post-metamórficos de cirripedios, registrados en el contenido estomacal de Chiton granosus.

Figure 2. Mean abundance ( \pm SD; $N=30$ ) of barnacle's cyprids and post-metamorphic stages, recorded in the gut content of Chiton granosus.

En la porción final del intestino (bolos fecales) de Ch. granosus se encontraron restos calcáreos procedentes de las placas de cirripedios adultos, larvas estado cypris (Fig. 3b), restos de ceramiales y estados post-metamórficos de Jehlius cirratus. De estos ítemes, las larvas cypris se observaron en un mayor número de individuos $(\mathrm{F} \%=67)$. Además, se observó en el $45 \%$ de los individuos analizados que algunas larvas cypris $(\mathrm{n}=15$ en total) presentaban signos de actividad vital (movimiento de sus apéndices) antes de la fijación de los individuos en formalina.
En la evaluación de la dieta de Ch. granosus cada dos meses de febrero a octubre de 2001, se determinó una alta frecuencia de cirripedios en estado de larvas cypris y estados post-metamórficos de $J$. cirratus, éstos se observaron con una frecuencia porcentual promedio para cinco meses de estudio de $96 \%( \pm 9,08 ;$ máx $=38$; mín $=1)$ y $78 \%( \pm 20,19$; máx = 28; mín =1), respectivamente (Fig. 4).

En el análisis del contenido estomacal de $C h$. granosus se registró un importante número de larvas y estados post-metamórficos de cirripedios, aun-

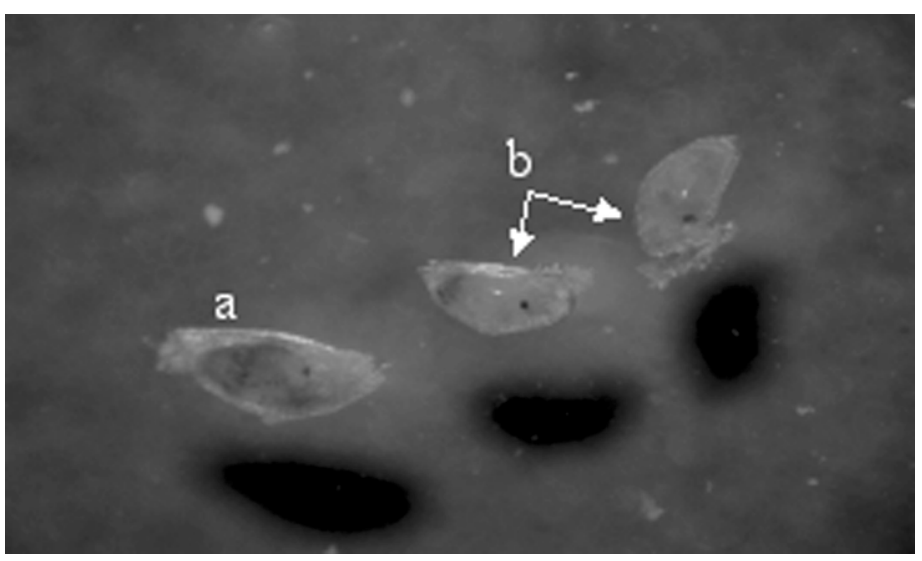

Figura 3. a) Larvas cypris de cirripedios observadas en el contenido estomacal, b) parte posterior del intestino (bolos fecales) de Chiton granosus.

Figure 3. a) Barnacle's cyprids larvae observed in the gut content, b) final portion of intestine (fecal pellet) of Chiton granosus. 


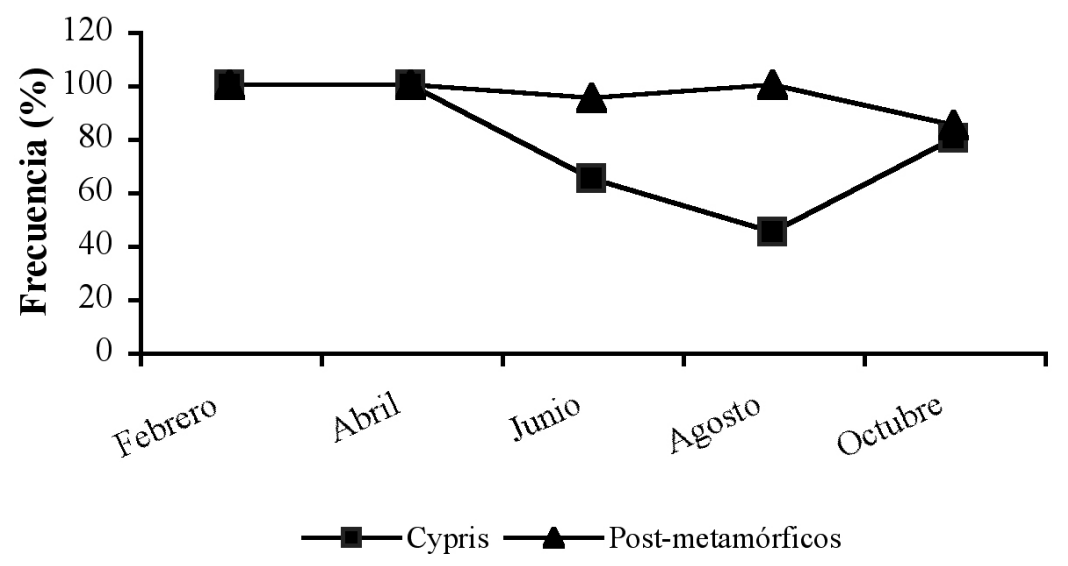

Figura 4. Frecuencia porcentual (F\%) de larvas cypris y estados post-metamórficos de cirripedios, registrados en el contenido estomacal de Chiton granosus $(\mathrm{N}=10)$ entre febrero y octubre de 2001 en el sector de Península Cavancha, Iquique.

Figure 4. Percent frequency ( $F \%$ ) of barnacle's cyprids and post-metamorphic stages, recorded in the gut content of Chiton granosus $(\mathbf{N}=10)$ between February and October 2001 in Península Cavancha, Iquique.

que también se encontraron larvas cypris en la porción final del intestino en un porcentaje de individuos superior al $60 \%$ sin daños estructurales aparentes. Esto último podría sugerir que los ácidos y enzimas digestivas de $C h$. granosus no produjeron daños significativos a estas larvas, más aún cuando algunas presentaron signos de actividad vital. Es probable entonces, que Ch. granosus no digiera estas larvas o que solo esté digiriendo efectivamente una baja cantidad, por lo que no serían el alimento principal de esta especie como lo señala Jara (1980, fide Moreno \& Jaramillo, 1983). En otro contexto, es importante considerar si estas larvas luego de atravesar el tracto digestivo de los chitones podrían asentarse efectivamente en el sustrato. Esto, en forma similar a como sucede con propágulos de algunas macroalgas que luego de pasar por el tracto digestivo de algunos moluscos herbívoros intermareales (incluyendo a $C h$. granosus), pueden asentarse y germinar en forma efectiva (Santelices \& Correa, 1985). No obstante, en las larvas cypris de cirripedios, se requiere de estudios experimentales posteriores que puedan determinar claramente su viabilidad post-ingestión.

En el caso de los estados post-metamórficos de cirripedios, aunque se observó una baja presencia en el tracto intestinal esto puede haber sido producto de una mayor asimilación en el tracto digestivo de Ch. granosus, o a que las ofertas en el ambiente no hayan sido tan altas como para verse reflejadas en el contenido estomacal e intestinal de esta especie. Así mismo, es muy probable que tanto larvas como estados post-metamórficos de cirripedios hayan sido ingeridos en forma accidental, por lo que las proporciones ingeridas dependerían directamente de las ofertas del ambiente lo cual requiere de confirmación posterior. Además, si la ingesta de postmetamórficos de cirripedios y de otros invertebrados es frecuente, como se observó en todos los meses de estudio, esto podría tener importantes consecuencias en relación a las características alimenticias de esta especie. Así por ejemplo, si dicha ingesta tiene un aporte nutricional importante en la dieta de Ch. granosus (por ej: micronutrientes), podría más bien considerarse a esta especie como un molusco omnívoro más que como un herbívoro estricto lo cual ha sido señalado anteriormente para otros chitones (Kaas \& van Belle, 1985).

La ingesta de cirripedios en los diferentes estados de desarrollo observados, podría tener importantes consecuencias en la abundancia y distribución de cirripedios en la zona intermareal, más aún dadas las altas densidades que Ch. granosus puede alcanzar en ciertas localidades de Chile central (Otaíza \& Santelices, 1985). Esto, debido a que se ha observado en otras latitudes que algunos moluscos herbívoros pueden afectar en forma significativa la recolonización de cirripedios mediante 
ingesta y remoción mecánica (bulldozing) de sus cypris y estados post-metamórficos (Connell, 1961; Dayton, 1971; Paine, 1981; Chan \& Williams, 2003).

\section{AGRADECIMIENTOS}

El autor desea agradecer al Biólogo Marino Sr. Guillermo Guzmán, por su apoyo durante el desarrollo del estudio y sus correcciones al manuscrito. Al Sr. Luis Balboa por sus correcciones iniciales del manuscrito. También se agradece el patrocinio del Departamento de Ciencias del Mar de la Universidad Arturo Prat, en cuyos laboratorios se realizó el presente estudio en el marco del seminario de investigación del autor.

\section{REFERENCIAS}

Connell, J.H. 1961. Effects of competition, predation by Thais lapillus and other factors on natural populations of the barnacle Balanus balanoides. Ecol. Monogr., 31: 61-104.

Chan, B.K. \& G.A. Williams. 2003. The impact of physical stress and molluscan grazing on the settlement and recruitment of Tetraclita species (Cirripedia: Balanomorpha) on a tropical shore. J. Exp. Mar. Biol. Ecol., 284: 1-23.

Dayton, P.K. 1971. Competition, disturbance and community organization: the provision and subsequent utilization of space in a rocky intertidal community. Ecol. Monogr., 41: 351-389.

Recibido: 24 mayo 2004; Aceptado: 23 marzo 2005
Hill, A.S. \& S.J. Hawkins. 1991. Seasonal and spatial variation of epilithic microalgal distribution and abundance and its ingestion by Patella vulgata on a moderately exposed rocky shore. J. Mar. Biol. Ass. U.K., 71: 403-423.

Kaas, P. \& R. van Belle. 1985. Monography of living chitons. Sprintg-Verlag, Berlin, 400 pp.

Moreno, C.A. \& E. Jaramillo. 1983. The role of grazers in the zonation of intertidal macroalgae of the Chilean coast. Oikos, 41: 73-76.

Otaíza, R.D. 1986. Patrones de distribución vertical de chitones y efecto de pastoreo de Chiton granosus en roqueríos intermareales de Chile central. En: B. Santelices (ed.). Simposio Internacional. Usos y funciones ecológicas de las algas marinas bentónicas. Monografías Biológicas, 4: 173 -190.

Otaíza, R.D. \& B. Santelices. 1985. Vertical distribution of chitons (Mollusca: Polyplacophora) in the rocky intertidal zone of central Chile. J. Exp. Mar. Biol. Ecol., 86: 229-240.

Paine, R.T. 1981. Barnacle ecology: is competition important? The forgotten roles of disturbance and predation. Paleobiology, 7: 553-560.

Santelices, B. \& J. Correa. 1985. Differential survival of macroalgae to digetion by intertidal herbivore molluscs. J. Exp. Mar. Biol. Ecol., 88: 183-191.

Santelices, B., J. Vásquez \& I. Meneses. 1986. Patrones de distribución y dietas de un gremio de moluscos herbívoros en hábitats intermareales expuestos de Chile central. En: B. Santelices (ed.). Simposio Internacional. Usos y funciones ecológicas de las algas marinas bentónicas. Monografías Biológicas, 4: 147-171. 